Abanico Veterinario. Enero-Diciembre 2021; 11:1-11. http://dx.doi.org/10.21929/abavet2021.43 Nota Corta. Recibido: 08/09/2021. Aceptado:01/12/2021. Publicado: 28/12/2021. Clave: e2021-64. https://www.youtube.com/watch?v=dnsmwSHMMZ8

\title{
Respuesta reproductiva en yeguas criollas tratadas con acetato de deslorelina
}

\author{
Reproductive response in creole mares treated with deslorelin acetate
}

\section{Lara-Castillo Jorge ${ }^{11 \mathrm{D}}$, Herrera-Camacho José ${ }^{1 \mathrm{ID}^{*}}$, Estrada-Coates Alejandro ${ }^{2 \mathrm{ID}}$, Bautista-Hernández Miguel $^{3 \mathrm{ID}}$, Gómez-Ramos Benjamin ${ }^{3 \mathrm{ID}}$}

${ }^{1}$ Instituto de Investigaciones Agropecuarias y Forestales-Universidad Michoacana de San Nicolás de Hidalgo. Posta Zootecnia km 9.5 Carretera Morelia-Zinapécuaro. Col. El Trébol. CP 58880.Tarímbaro,

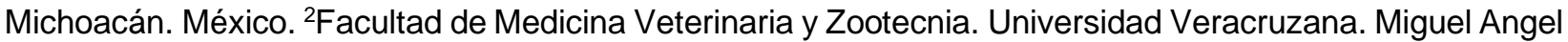
de Quevedo s/n esquina Yáñez. Colonia Unidad Veracruzana CP. 91710. Veracruz, Veracruz. México. ${ }^{3}$ Facultad de Medicina Veterinaria y Zootecnia-Universidad Michoacana de San Nicolás de Hidalgo. Posta Zootecnia km 9.5 Carretera Morelia-Zinapécuaro. Col. El Trébol. CP 58880. Tarímbaro, Michoacán. México. *autor de correspondencia: Herrera-Camacho José. E-mail: jorge.barzilai.lara.castillo@umich.mx, jose.camacho@umich.mx, aestradatc@yahoo.com.mx, mbautista@umich.mx, benjamin.gomez@umich.mx

\section{RESUMEN}

El efecto del acetato de deslorelina sobre la respuesta reproductiva fue evaluado en yeguas criollas en el Estado de Michoacán. Diez yeguas tratadas intramuscularmente y distribuidas en un Grupo control (GC; $\mathrm{n}=5$ ) $1 \mathrm{ml}$ de agua destilada como placebo y el grupo experimental (GD; $\mathrm{n}=5$ ) $1.5 \mathrm{mg}$ del acetato de deslorelina. Cuando el folículo alcanzó los $35 \mathrm{~mm}$ de diámetro, las yeguas se trataron en función del grupo asignado. La actividad ovárica fue monitoreada cada 24 h, y se determinó la incidencia (\%) de folículos hemorrágicos anovulatorios (HAFs), tasa de ovulación (\%), crecimiento folicular por día ( $\mathrm{mm})$, diámetro folicular a la ovulación $(\mathrm{mm})$, y tiempo a la ovulación $(\mathrm{h})$. La incidencia de HAFs fue superior $\left(\mathrm{X}^{2}=3.83\right)$ en el GC que en GD (25.58\% vs. 5.8\%, respectivamente). La tasa de ovulación fue superior $\left(X^{2}=4.76\right)$ en GD que en GC (94.11\% vs $74.41 \%$, respectivamente). La administración de la hormona, no afecto ( $>0.05)$ el crecimiento folicular por día ni el diámetro folicular, pero la ovulación se presentó $(p<0.01)$ a las $39.45 \pm 2.95$ h y $89.47 \pm 3.62 \mathrm{~h}$ en el GD y GC, respectivamente. El acetato de deslorelina redujo la incidencia de HAFs e incrementó la tasa de ovulación.

Palabras clave: yeguas criollas, acetato de deslorelina, folículos hemorrágicos anovulatorios.

\begin{abstract}
The effect of deslorelin acetate on reproductive response was evaluated in creole mares in the State of Michoacán. Ten mares were treated intramuscularly and distributed in a Control group (CG; $n=5) 1 \mathrm{ml}$ of distilled water as a placebo and the experimental group (DG; $n=5) 1.5 \mathrm{mg}$ of deslorelin acetate. When the follicle reached $35 \mathrm{~mm}$ in diameter, the mares were treated according to the assigned group. The ovarian activity was monitored every $24 \mathrm{~h}$. The incidence (\%) of anovulatory hemorrhagic follicles (HAFs), ovulation rate $(\%)$, follicular growth per day $(\mathrm{mm})$, follicular diameter at ovulation $(\mathrm{mm})$, and time to ovulation $(\mathrm{h})$ were determined. The incidence of HAFs was higher $(X 2=3.83)$ in the CG than in DG $(25.58 \%$ vs. $5.8 \%$, respectively). The ovulation rate was higher $(X 2=4.76)$ in DG than in CG $(94.11 \%$ vs. $74.41 \%$, respectively). The hormone administration did not affect $(p>0.05)$ the follicular growth per day or the follicular diameter, still, ovulation occurred $(p<0.01)$ at $39.45 \pm 2.95 \mathrm{~h}$ and $89.47 \pm 3.62 \mathrm{~h}$ in the DG and CG, respectively. deslorelin acetate reduced the incidence of HAFs and increased the ovulation rate.
\end{abstract}

Keywords: Creole mares, deslorelin acetate, anovulatory hemorrhagic follicles. 


\section{INTRODUCCIÓN}

La anovulación es una de las principales causas de infertilidad en diferentes hembras de animales domésticos, dado que retrasa la oportunidad de que la hembra quede gestante. Uno de los tipos de disfunción observados en yeguas es la presencia de folículos anovulatorios persistentes (FAP, McCue \& Squires, 2002), folículos hemorrágicos anovulatorios (HAFs; Cuervo-Arango \& Newcombe, 2012), o también llamados folículos luteinizados (LUF, Bashir et al., 2016). Estas estructuras se presentan cuando un folículo preovulatorio que tuvo un desarrollo normal, no logra romperse u ovular y el antro folicular se llena de sangre, proveniente del rompimiento de vénulas y arteriolas que irrigan las células de la granulosa. La presencia de HAFs/LUF, es la forma más común de anovulaciones en la yegua. Estudios previos han reportado una incidencia de $22.2 \%$ en yeguas en el Reino Unido (Lefranc \& Allen, 2003), y de $24 \%$ durante la temporada reproductiva en yeguas cuarto de milla en Estados Unido de América (Ginther et al., 2008); no obstante, también se ha reportado la presencia de HAFs durante la época de transición; es este sentido, McCue \& Squires (2002) determinaron, una incidencia de $8.2 \%$ de HAFs, en 1845 ciclos estrales registrados durante un periodo de 5 años en la Universidad de Colorado, USA.

Una yegua con presencia de HAFs manifiesta signos típicos del estro de manera repetida y prolongada; sin embargo; no logra liberar el ovocito, por lo cual no existirá gestación. Se ha reportado que dichos folículos anovulatorios afectan negativamente la tasa de ovulación (Gerard \& Robin, 2019).

Adicionalmente, un HAF, por lo general sufrirá un proceso de luteinización, es decir, desarrolla de tejido lúteo vascularizado ante la ausencia de ovulación, el antro folicular se llena de sangre, el cual se aprecia con focos ecogénicos y hebras o coágulos que dan la apariencia de fibrina (Jacob et al., 2009).

La presencia de HAFs, se ha asociado con algunos factores de riesgo, tales como el momento en la temporada reproductiva, edad y la utilización de sustancias exógenas y hormonales (Ginther et al., 2008).

Los HAFs generalmente deben identificarse mediante la ecografía, y se han utilizado hormonas para incrementar la liberación o concentración de hormona luteizante, entre ellas se encuentran la Gonadotropina Coriónica humana (hCG), con resultados de $88.3 \%$ de ovulación dentro de las primeras 48 horas de aplicada (McCue et al., 2007) y el Acetato de deslorelina, un análogo de la $(\mathrm{GnRH})$, el cual ha presentado una efectividad de $90.1 \%$ de ovulación durante las primeras 48 horas (McCue et al., 2007). En este mismo sentido, Finan et al. (2016), determinaron que la utilización de deslorelina en folículos de tamaño $\geq 30 \mathrm{~mm}$ mostraron una respuesta significativa con un $93.75 \%$ de ovulación. En México se han realizado pocos estudios para determinar la incidencia fallas anovulatorias en las yeguas. Es posible que ante su presencia el técnico administre en algunos casos hormonas exógenas para su tratamiento, y en otros casos se dejen pasar hasta que la yegua por sí sola presenta una ovulación efectiva. 
El objetivo del presente estudio fue evaluar la respuesta reproductiva en yeguas criollas tratadas con acetato de deslorelina.

\section{MATERIAL Y MÉTODOS}

Área de estudio: el presente estudio se realizó durante los meses de abril-agosto de 2020, en la comunidad de Santa Clara de Valladares del municipio de Tocumbo, Michoacán, México; localizado al Oeste del estado a $102^{\circ} 31^{\prime} 19^{\prime \prime}$ de Longitud Oeste y $19^{\circ} 42^{\prime} 10^{\prime \prime}$ de Latitud Norte, a una altitud de $1604 \mathrm{msnm}$, precipitación pluvial promedio de 800-1300 mm por año, con clima subhúmedo con lluvias en verano y una temperatura que oscila entre los $16-26^{\circ} \mathrm{C}$ (INEGI, 2009).

Animales: se utilizaron 10 yeguas criollas, con una edad entre 3 y 11 años, peso vivo entre 350 a 450 kilogramos, alzada a la cruz entre de 1.55-1.65 metros y una condición corporal de 4 a 6 puntos en la escala de Henneke (1984); ninguna de ellas estaba amamantando y tampoco tenían historial de problemas reproductivos previos. Las yeguas fueron alimentadas con una misma dieta, tomando como base el $8 \%$ del peso vivo de la hembra, considerando una concentración de 80:20 de forraje y concentrado, respectivamente. El forraje fue heno de avena y el alimento comercial como concentrado. El agua para bebida estuvo disponible ad libitum.

Tratamientos hormonales: previo al inicio del experimento las yeguas fueron sometidas a una revisión ecográfica diagnóstica con un equipo de ultrasonido transrectal de 7.5 $\mathrm{MHz}$, para conocer su estado reproductivo, ovárico y uterino. Todas las hembras fueron tratadas con una dosis de $5 \mathrm{mg}$ de prostaglandina $\mathrm{F}_{2 \alpha}\left(\mathrm{PGF}_{2 \alpha}\right.$, Dinoprost, Lutalyse, Zoetis $\mathrm{NZ}^{\circledR}$ ), para sincronizar el ciclo estral mediante la lisis del cuerpo lúteo, e iniciar el desarrollo de la fase folicular en un mismo tiempo y obtener condiciones ováricas homogéneas.

Posteriormente las yeguas fueron distribuidas en dos grupos: Grupo control (GC; n=5), el cual recibió la aplicación por vía intramuscular (im) de $1 \mathrm{ml}$ de agua destilada como placebo, y el grupo experimental (GD; $n=5)$, el cual recibió la aplicación, im, de 1.5 mg de la hormona clorhidrato de deslorelina. En ambos grupos la aplicación del placebo o la hormona se realizó cuando el folículo dominante alcanzó un diámetro $\geq 35 \mathrm{~mm}$. En total se registraron 77 ciclos estrales, 43 en el GC y 34 en el GD.

Las yeguas que presentaron ovulación y desarrollo de un cuerpo lúteo funcional alrededor del día 9 del ciclo estral recibieron una dosis de $5 \mathrm{mg}$ (im) de PGF $2 \alpha$ (Dinoprost, Lutalyse, Zoetis $\mathrm{NZ}^{\circledR}$ ) con la intención de reiniciar la dinámica folicular.

Revisión ovárica: la revisión ovárica de las yeguas de ambos grupos se realizó con un ultrasonido (US) de $7.5 \mathrm{Mhz}$ (Sonoescape S23, USA ${ }^{\circledR}$ ), a intervalos de $24 \mathrm{~h}$ para 
determinar la tasa de crecimiento folicular por día y previo al momento de la ovulación, la revisión ovárica se realizó a intervalos de $6 \mathrm{~h}$.

La incidencia (\%) de folículos hemorrágicos anovulatorios, se estableció cuando a la observación con el US un folículo mostraba signos de hemorragia interna formando el estigma folicular, pero sin que la ovulación tuviera lugar.

Para evaluar la eficiencia reproductiva se incluyeron los siguientes parámetros: el crecimiento folicular por día, diámetro folicular a la ovulación, tiempo a la ovulación después de la aplicación de la hormona y la tasa de ovulación.

El crecimiento folicular por día $(\mathrm{mm})$, se evaluó colocando el cursor del US en forma de cruz desde los bordes superior e inferior y derecho e izquierdo de cada folículo $\geq 35 \mathrm{~mm}$, para registrar el diámetro promedio en cada día de evaluación; el crecimiento folicular por día se calculó restando el diámetro actual al del día previo.

Una vez que se observó el estigma en el folículo de Graff, la revisión ecográfica se realizó a intervalos de $6 \mathrm{~h}$, registrando el diámetro a la ovulación $(\mathrm{mm})$ y el tiempo de ovulación (h), el cual fue considerado como el tiempo transcurrido entre la aplicación de la hormona hasta el rompimiento folicular, y se confirmó mediante la observación de la fosa ovulatoria hemorrágica y con la aparición de uno o más cuerpos lúteos.

Por otra parte, la tasa de ovulación (\%) fue considerada como número total de folículos que lograron la ovulación en cada uno de los ciclos estrales evaluados en las yeguas de ambos grupos, multiplicado por 100 y dividido entre el número de folículos dominantes que desarrollaron sobre la superficie del ovario.

Análisis estadístico: los resultados obtenidos de las variables incidencia (\%) de folículos anovulatorios y tasa de ovulación (\%), fueron sometidos a una prueba de $X^{2}$, en un cuadro de contingencia $2 \times 2$. El crecimiento folicular por día $(\mathrm{mm})$, diámetro folicular a la ovulación $(\mathrm{mm})$ y tiempo $(\mathrm{h})$ a la ovulación, fueron evaluados mediante una prueba de medidas repetidas en un modelo mixto, donde la yegua fue el efecto aleatorio, el tratamiento el efecto fijo y el ciclo estral de cada yegua como medida repetida. La comparación entre tratamientos se realizó mediante la prueba de mínimos cuadrados. El valor de significancia considerado fue $\mathrm{p}<0.05$ y todos los análisis se realizaron en el paquete estadístico SAS (SAS, 2013).

\section{RESULTADOS}

La incidencia de folículos anovulatorios fue de $25.58 \%$ (11/43 estros observados) en yeguas del GC; mientras que en el GD fue de $5.8 \%$ (2/34 estros tratados). Las diferencias observadas entre ambos grupos fueron estadísticamente significativas $\left(X^{2}=3.83\right)$.

El crecimiento folicular por día en yeguas criollas del estado de Michoacán no mostró diferencias ( $p>0.05$ ) entre los grupos de yeguas, observando un crecimiento diario de $2.15 \pm 0.19 \mathrm{~mm}$ para el GC y de $2.42 \pm 0.16 \mathrm{~mm}$ en el GD. 
El diámetro folicular a la ovulación no mostró diferencias significativas ( $p>0.05$ ) entre los grupos estudiados, observando un promedio folicular de $44.08 \pm 0.63 \mathrm{~mm}$ y $44.94 \pm 0.78$ $\mathrm{mm}$ en yeguas del GD y GC, respectivamente.

El tiempo (h) a la ovulación fue de $39.45 \pm 2.95 \mathrm{~h}$ para el GD, mientras que para el GC fue de $89.47 \pm 3.62$ h. Las diferencias observadas entre ambos grupos fueron estadísticamente significativas $(p<0.01)$ y represento una diferencia de $50 \mathrm{~h}$ a favor del GD.

La tasa de ovulación fue de $74.41 \%$ (32/43 ciclos estrales observados) y de $94.11 \%$ (32/34 ciclos estrales), en el GC y GD respectivamente; lo que indica un efecto favorable del acetato de deslorelina $\left(X^{2}=4.76\right)$.

\section{DISCUSIÓN}

La incidencia de HAFs observada en el presente estudio fue similar a lo reportado por Ginther et al. (2008), quienes observaron un $24 \%$ durante la temporada reproductiva en yeguas cuarto de milla. En el mismo sentido, Lefranc y Allen (2003), reportaron una incidencia de HAFs $22.2 \%$ en yeguas. Por el contrario, otros autores (McCue y Squires, 2002) observaron una incidencia de HAFs menor al 10\%, durante la temporada de transición.

Otros estudios, como los realizados por Cuervo-Arango \& Newcombe (2010), observaron un efecto de la edad sobre la incidencia de HAFs de $13.1 \%$ en yeguas de 6-10 años; mientras que en yeguas mayores de 10 años, la incidencia fue del $24 \%$, lo que indica que la temporada reproductiva y edad de la yegua, pueden ser factores predisponentes a la formación de folículos hemorrágicos anovulatorios (Ginther et al., 2008). Estudios previos en México (López et al., 2010), reportaron la presencia de folículos anovulatorios; sin embargo, no se indica la incidencia observada.

La yegua es una especie con reproducción estacional y tiene un periodo de transición ovárica en primavera, entre el anestro de invierno y la renovación de su ciclicidad durante la estación reproductiva. Durante la transición de primavera las concentraciones de LH son bajas, posiblemente causadas por el efecto de la melatonina bloqueando la hormona liberadora de gonadotropinas (Satue \& Gordon, 2020). Lo anterior explica por que las yeguas pueden presentar ondas foliculares anovulatorias, que pueden incluso, alcanzar el tamaño de los folículos preovulatorios y convertirse en HAFs con mayor predisposición (Watson \& Al-Zi'abi, 2002).

Con respecto a la edad, en la yegua como en los demás animales domésticos existen cambios degenerativos asociados con los HAFs, se da a partir de la alteración en la síntesis de la enzima glutatión peroxidasa y enzima encargada de la eliminación de especies reactivas a oxígeno; si esta hormona se encuentra restringida, los ovocitos y las células de la pared del folículo pierden su capacidad para responder a la colagenasa, limitando el efecto de la hormona LH sobre las células de la teca interna, permitiendo que solo adelgace las paredes del folículo (Ginther \& Beg, 2011), hecho que aún con el efecto 
físico de la ovulación no es capaz de romper la pared folicular y liberar el material genético, dando origen así al folículo anovulatorio (Morel et al., 2005).

El uso de agentes exógenos y hormonales con intención de inducir a la ovulación como la LH o análogos del $\mathrm{GnRH}$, han tenido como resultado alteraciones ovulatorias y/o formación de HAFs (Cuervo-Arango \& Newcombe, 2010). Estudios previos realizados por Schauer et al. (2013), planteaban que la hormona LH no interrumpía los patrones de crecimiento folicular ni la ovulación; sin embargo, sí alteró los factores del fluido folicular que podrían interrumpir la maduración de los ovocitos o folículos (Burden et al., 2015). La hormona LH permite que a través de la activación de la matriz de las metaloproteinasas y la activación de plasminógenos, se presente la remodelación tisular del folículo, ya que a mayor concentración de $\mathrm{E}_{2}$ y LH se incrementa la colagenasa III en el estroma ovárico y disminuye la colagenasa I en la periferia del folículo dominante (Smok \& Rojas, 2010). Esta hormona también tiene una participación estrecha con las prostaglandinas que se encargan de provocar la ovulación con las COX II (CuervoArango \& Martínez-Boví, 2016). La producción de LH, previo a la ovulación es la encargada de luteinizar estos folículos, permitiendo que haya una infiltración de células grasas a través de la pared del folículo y de esta manera se luteiniza, hecho que bloquea la salida del ovocito.

En el presente estudio, la aplicación del acetato de deslorelina, fue eficaz para reducir la incidencia de HAFs en yeguas criollas en el estado de Michoacán; es posible que su efectividad se debió a su efecto promotor en la amplitud y frecuencia de los pulsos de liberación de la LH hipofisiaria, provocando la activación de la colagenasa y por ende desencadeno la ovulación, evitando la formación de HAFs. (Squires et al., 1994).

Respecto al crecimiento folicular por día, los resultados encontrados en este estudio son similares a los reportados por Donadeu \& Pedersen (2008); no obstante, McCue (2007a) y McCue (2007b), quienes observaron un crecimiento folicular diario que oscilo de 2.1 a $4.3 \mathrm{~mm}$ por día. Las alteraciones en dicho crecimiento implican que el momento preciso de la ovulación no sea estimado con certeza, ya que su comportamiento es impredecible y pueden ovular de manera espontánea, por lo cual requieren de una mayor atención con respecto al momento de la ovulación, debiendo ser monitoreadas ecográficamente de manera frecuente (Dordas-Perpinyà et al., 2020).

Uno de los factores frecuentemente utilizados como un criterio práctico para predecir la ovulación en la yegua, es el diámetro folicular, dado que es un método simple y relativamente fácil de monitorear cuando se cuenta con la experiencia en el uso del ultrasonido (Cuervo-Arango \& Newcombe, 2008). Los trabajos realizados han evidenciado un rango de 34 a $70 \mathrm{~mm}$ en el diámetro de folículos preovulatorios $24 \mathrm{~h}$ previas a la ovulación, donde la raza de la yegua, condición corporal y la época del año, juegan un papel importante; por ello se hace uso de tratamientos hormonales y poder predecir el momento oportuno para dar servicio a la yegua e incrementar la eficiencia reproductiva. 
Al respecto, Cuervo-Arango \& Newcombe (2008) encontraron que el diámetro del folículo preovulatorio disminuye cuando se aplica un tratamiento hormonal respecto al diámetro folicular de ovulación espontánea o natural. Estos autores reportaron un diámetro folicular de $38.82 \pm 0.83$ y $44.18 \pm 1.22$, tratadas con $1500 \mathrm{UI}$ de $\mathrm{hCG}$ en yeguas con ovulación espontánea, respectivamente. También observaron un diámetro folicular menor en yeguas tratadas con $\mathrm{PGF}_{2 \alpha}(39.42 \pm 1.77$ vs $47.77 \pm 2.00)$ con un implante de $2.6 \mathrm{mg}$ de deslorelina ( $34.25 \pm 0.65$ vs $40.92 \pm 1.38$ ) con respecto a la ovulación natural.

El uso de los diferentes compuestos hormonales ha supuesto un diámetro folicular al momento de la ovulación similar al observado en el presente estudio. Dolezel et al. (2012), reportaron un diámetro folicular entre 44 y 48 mm. Dordas-Perpinyà et al. (2020), no reportaron diferencias significativas en el diámetro folicular de yeguas tratadas con acetato de buserelina (6 mg) o con eCG (1500 UI); éstas alcanzaron un diámetro folicular promedio de $40.98 \pm 0.55 \mathrm{~mm}$ y de $41.45 \pm 0.59 \mathrm{~mm}$, respectivamente. En este mismo estudio se realizó el tratamiento de yeguas con $3 \circ 6 \mathrm{mg}$ de acetato de buserelina y tampoco se reportaron diferencias significativas entre ambos grupos, encontrando un diámetro folicular de $41.64 \pm 3.63 \mathrm{~mm}$ y $41.95 \pm 3.79 \mathrm{~mm}$, respectivamente.

De igual manera Dordas-Perpinyà et al. (2020), evaluaron la eficacia de diferentes tratamientos hormonales en yeguas e incluyeron $0.1 \mathrm{mg}$ de triptorelina, $1500 \mathrm{UI}$ de hCG, 3,2 y $1 \mathrm{mg}$ de acetato de buserelina y observaron el diámetro folicular por tratamiento a la ovulación de $42.1 \pm 2.8,43.1 \pm 4.2,42.5 \pm 2.9,42.6 \pm 2.8,43.7 \pm 3.8 \mathrm{~mm}$, respectivamente; siendo diferentes $(\mathrm{p}<0.01)$ con respecto al diámetro folicular en ovulación espontánea, el cual fue de $45.6 \pm 5.3 \mathrm{~mm}$, media superior a los tratamientos hormonales e incluso ligeramente superior a lo observado en nuestro estudio.

Algunos autores (Cuervo-Arango \& Newcombe, 2008) han señalado que los tratamientos hormonales para inducir a la ovulación disminuyen el tamaño del folículo preovulatorio, en comparación con la ovulación natural; y aunque existe poca información al respecto, es posible que el incremento en la concentración de LH, ya sea por la administración de agonistas de GnRH como deslorelina, buserelina u otros, o bien por análogos como hCG, provocan una disminución de la actividad hipofisiaria para la producción de hormona folículo estimulante, limitando el crecimiento folicular, permitiendo la ovulación más temprana que cuando se mantiene el crecimiento folicular natural.

El tiempo transcurrido desde la aplicación de la hormona deslorelina y otros análogos del $\mathrm{GnRH}$ reducen significativamente el tiempo de ovulación. Estudios previos (Miki et al., 2016), con yeguas con un diámetro folicular igual o mayor que $35 \mathrm{~mm}$, han reportado un incremento en los niveles de LH dentro de las primeras 6 a $24 \mathrm{~h}$ de aplicación en yeguas de tiro pesado, en contraste con los picos de LH reportados 1 a 2 días después de la ovulación, según los patrones normales reportados para yeguas (Meinecke et al., 1987). La liberación anticipada de LH disminuye la posibilidad de luteinizar el folículo preovulatorio e infiere positivamente en la disminución de folículos anovulatorios. 
Los resultados obtenidos en el presente estudio para la tasa de ovulación son similares a los reportados por Finan et al. (2016), quienes trabajaron con yeguas nativas de Australia, observando un $93.7 \%$ de ovulación, utilizando un implante subcutáneo de 1.25 mg de deslorelina.

Los resultados obtenidos en el presente estudio con respecto a la ovulación, son similares a los obtenidos en diferente posición geográfica y raza de las yeguas, hecho que podía predecirse, ya que la hormona y sus análogos demostraron resultados positivos, comprobando su respuesta terapéutica sobre los gonadótrofos por su efecto análogo de la LH (D’Oliveira et al., 2019).

\section{CONCLUSIÓN}

La administración de la hormona acetato de deslorelina mejoró la respuesta reproductiva en yeguas criollas del estado de Michoacán, al reducir la incidencia de HAFs, incrementar la tasa de ovulación y reducir el tiempo a la ovulación.

\section{LITERATURA CITADA}

BASHIR ST, Gastal MO, Tazawa SP, Tarso SGS, Hales DB, Cuervo-Arango J, Baerwald AR, Gastal EL. 2016. The mare as a model for luteinized unruptured follicle syndrome: intrafollicular endocrine milieu. Reproduction. 151 (3): 271-283.

https://doi.org/10.1530/REP-15-0457

BURDEN CA, McCue PM, Ferris RA. 2015. Effect of cloprostenol administration on interval to subsequent ovulation and anovulatory follicle formation in quarter horse mares. Journal of Equine Veterinary Science. 35(6): 531-535.

https://doi.org/10.1016/j.jevs.2015.02.011

CUERVO-ARANGO J, Martínez-Boví R. 2016. The role of PGE2 and PGF2a in follicle wall rupture and their implications in the development and treatment of luteinized unruptured follicles. Pferdeheilkunde Equine Medicine. 32(1):54-56.

https://doi.org/10.21836/pem20160110

CUERVO-ARANGO J, Newcombe J. 2012. Ultrasound characteristics of experimentally induced luteinized unruptured follicles (LUF) and naturally occurring hemorrhagic anovulatory follicles (HAF) in the mare. Theriogenology. 77(3): 514-524.

https://doi.org/10.1016/j.theriogenology.2011.08.026

CUERVO-ARANGO J, Newcombe JR. 2008. Repeatability of preovulatory follicular diameter and uterine edema pattern in two consecutive cycles in the mare and how they are influenced by ovulation inductors. Theriogenology. 69(8): 681-687. https://doi.org/10.1016/j.theriogenology.2007.11.019 
CUERVO-ARANGO J, Newcombe JR. 2010. Risk factors for the development of haemorrhagic anovulatory follicles in the mare. Reproduction in Domestic Animals. 45(7): 473-480. https://doi.org/10.1111/j.1439-0531.2008.01260.x

D' OLIVEIRA SN, Canuto L, Segabinazzi LGTM, Dell'Aqua JJA, Papa P, Fonseca M, Ribeiro FADL, Papa F. 2019. Histrelin acetate-induced ovulation in Brazilian Northeastern jennies (Equus asinus) with different follicle diameters. Theriogenology. 136 (1):95-100. https://doi.org/10.1016/j.theriogenology.2019.06.037

DOLEZEL R, Ruzickova K, Maceckova G. 2012. Growth of the dominant follicle and endometrial folding after administration of $\mathrm{hCG}$ in mares during oestrus. Veterinarni Medicina. 57 (1):36-41. https://doi:10.17221/4970-VETMED

DONADEU F, Pedersen H. 2008. Follicle development in mares. Reproduction in Domestic Animals. 43(7): 224-231. https://doi.org/10.1111/j.1439-0531.2008.01166.x

DORDAS-PERPINYÀ M, Normandin L, Dhier T, Terris H, Cochard A, FrilleyC, Huiban F, Bruyas JF. 2020. Single injection of triptorelin or buserelin acetate in saline solution induces ovulation in mares the same as a single injection of hCG. Reproduction in Domestical Animals. 55(9):374-383. https://doi.org/10.1111/rda.13632

FINAN SA, Lamkinb EL, McKinnon AO. 2016. Comparative efficacy of BioRelease Deslorelin $\AA$ injection for induction of ovulation in oestrus mares: a field study. Australian Veterinary Journal. 94(3):338-340. https://doi.org/10.1111/avj.12478

GERARD N, Robin E. 2019. Cellular and molecular mechanisms of the preovulatory follicle differenciation and ovulation: What do we know in the mare relative to other species. Theriogenology. 130 (1):163-176.

https://doi.org/10.1016/j.theriogenology.2019.03.007

GINTHER OJ, Beg MA. 2011. Hormone concentration changes temporally associated with the hour of transition from preluteolysis to luteolysis in mares. Animal Reproduction Science. 129(6): 67-72. https://doi.org/10.1016/j.anireprosci.2011.09.013

GINTHER OJ, Gastal EL, Gastal MO, Jacob JC, Beg MA. 2008. Induction of haemorrhagic anovulatory follicles in mares. Reproduction, Fertility and Development. 20(5): 947-954. https://doi.10.1071/rd08136 
HENNEKE D, Potter G, Kreider J. 1984. Body condition during pregnancy and lactation and reproductive efficiency of mares. Theriogenology. 21(6):897-909.

https://doi.org/10.1016/0093-691x(84)90383-2

INEGI. 2009. Instituto Nacional de Estadística Geografía e Informática. Prontuario de información geográfica municipal de los Estados Unidos Mexicanos. Tocumbo, Michoacán de Ocampo. Clave geoestadística 16095.

http://www3.inegi.org.mx/contenidos/app/mexicocifras/datos_geograficos/16/16095.pdf

JACOB JC, Gastal EL, Gastal MO, Carvalho GR, Beg M A, Ginther O J. 2009. Temporal relationships and repeatability of follicle diameters and hormone concentrations within individuals in mares. Reproduction in Domestic Animals. 44(7): 92-99.

https://doi.org/10.1111/j.1439-0531.2007.01003.x

LEFRANC AC, Allen WR. 2003. Incidence and morphology of anovulatory haemorrhagic follicles in the mare. Pferdeheilkunde Equine Medicine. 19(6): 611-612.

https://doi.org/10.21836/pem20030607

LÓPEZ-PÉREZ LM, Zarco-Quintero L, Boeta-Acosta AM. 2010. Inducción de la actividad ovárica en yeguas criollas con un programa de fotoperiodo artificial en la latitud $19^{\circ} 9^{\prime} \mathrm{N}$. Veterinaria México. 41(2): 89-100.

http://www.scielo.org.mx/scielo.php?script=sci_arttext\&pid=S0301$50922010000200002 \& \operatorname{lng}=e s \&$ Ing $=e s$

MCCUE PM, Magee C, Gee EK. 2007. Comparison of compounded deslorelin and hCG for induction of ovulation in mares. Journal of Equine Veterinary Science. 27(3): 58- 61. https://doi.org/10.1016/j.jevs.2006.12.003

MCCUE PM, Squires EL. 2002. Persistent anovulatory follicles in the mare. Theriogenology. 58(5): 541-543. https://doi.org/10.1016/S0093-691X(02)00769-0

MCCUE PM. 2007a. Ovarian abnormalities. In Current therapy in equine reproduction. Saunders Elsevier. USA. ISBN 13: 978-0-7216-0252-3. 13(5): 87-92. https://doi.org/10.1016/B978-0-7216-0252-3.50017-5

MCCUE PM. 2007b. Ovulation failure. In Current therapy in equine reproduction. Saunders Elsevier. USA. ISBN 13: 978-0-7216-0252-3. 12(3): 83-86. https://doi.org/10.1016/B978-0-7216-0252-3.50016-3 
MEINECKE B, Gips H, Meinecke TS. 1987. Progestagen, androgen and estrogen levels in plasma and ovarian follicular fluid during the oestrous cycle of the mare. Animal Reproduction Science. 12(4):255-265. https://doi.org/10.1016/0378-4320(87)90097-2

MIKI W, Oniyama H, Takeda N, Kimura Y, Haneda S, Matsui M, Taya K, Nambo Y. 2016. Effects of a single use of the $\mathrm{GnRH}$ analog buserelin on the induction of ovulation and endocrine profiles in heavy draft mares. Journal of Equine Science. 27(4):149-156. https://doi.10.1294/jes.27.149

MOREL MCD, Newcombe JR, Swindlehurst JC. 2005. The effect of age on multiple ovulation rates, multiple pregnancy rates and embryonic vesicle diameter in the mare. Theriogenology. 63 (9): 2482-2493. https://doi.org/10.1016/j.theriogenology.2004.09.058

SATUÉ K, Gardon JC. 2020. Physiological and clinical aspects of the endocrinology of the estrous cycle and pregnancy in mares. In Animal Reproduction in Veterinary Medicine. Aral F, Payan-Carreira R, Quaresma M. Ed. IntechOpen, London, United Kingdom. ISBN: 978-1-83881-937-8. https://doi.org/10.5772/intechopen.90387 https://www.intechopen.com/chapters/70381

SCHAUER S, Guillaume D, Decourt C, Watson E, Briant C, Donadeu F. 2013. Effect of luteinizing hormone overstimulation on equine follicle maturation. Theriogenology. 79(3):409-416. https://doi.org/10.1016/j.theriogenology.2012.08.017

SMOK SC, Rojas RM. 2010. Follicular-stromal interaction in the mare ovary during the reproductive cycle. International Journal of Morphology. 28(3):697-701. http://dx.doi.org/10.4067/S0717-95022010000300007

SQUIRES E, Moran D, Farlin M, Jasko D, Keefe T, Meyers S, Figueiredo E, McCue P, Jochle W. 1994. Effect of dose of $\mathrm{GnRH}$ analog on ovulation in mares. Theriogenology. 41(3): 757-769. https://doi.org/10.1016/0093-691x(94)90185-।

WATSON E, Al-Zi'abi M. 2002. Characterization of morphology and angiogenesis in follicles of mares during spring transition and the breeding season. Reproduction. 20(7):227-234. https://doi.org/10.1530/rep.0.1240227

SAS. Statistical Analysis System. SAS Institute. 2012. JMP Statistics and Graphics Guide. Version 4.0. SAS Institute, Cary, NC. ISBN: 978-1-60764-599-3

http://www.sas.com/en_us/software/analytics/stat.html\# 